\title{
Discovering novel neuroactive drugs through high-throughput behavior-based chemical screening in the zebrafish
}

\section{Giancarlo Bruni, Parth Lakhani and David Kokel*}

Cardiovascular Research Center, Massachusetts General Hospital and Harvard Medical School, Charlestown, MA, USA

\section{Edited by:}

Gul Erdemli, Novartis, USA

\section{Reviewed by:}

Rahman M. Mizanur, US Army Medical Research Institute of Infectious Diseases, USA Jason Rihel, University College London, UK

Fabrizio Serluca, Novartis Institutes for Biomedical Research, USA

\section{*Correspondence:}

David Kokel, Cardiovascular Research Center, Massachusetts General

Hospital and Harvard Medical School,

Charlestown, MA, USA

e-mail:dkokel@cvrc.mgh.harvard.edu
Most neuroactive drugs were discovered through unexpected behavioral observations. Systematic behavioral screening is inefficient in most model organisms. But, automated technologies are enabling a new phase of discovery-based research in central nervous system (CNS) pharmacology. Researchers are using large-scale behavior-based chemical screens in zebrafish to discover compounds with new structures, targets, and functions. These compounds are powerful tools for understanding CNS signaling pathways. Substantial differences between human and zebrafish biology will make it difficult to translate these discoveries to clinical medicine. However, given the molecular genetic similarities between humans and zebrafish, it is likely that some of these compounds will have translational utility. We predict that the greatest new successes in CNS drug discovery will leverage many model systems, including in vitro assays, cells, rodents, and zebrafish.

\section{Keywords: behavior-based drug discovery, zebrafish, phenomics, antipsychotics, screening}

\section{INTRODUCTION}

At a recent course on neurotherapeutic drug discovery (sponsored by the National Institutes of Health) the keynote speaker joked that if he were trying to be rational, he would not be trying to discover neuroactive drugs. His point was that drug discovery is often much more empirical than rational. Someday, when researchers understand the biochemical mechanisms of psychiatric disease, it may be possible to discover neuroactive drugs based on rational therapeutic hypotheses. Until then, phenotypic assays provide an alternative approach. Behavior-based drug discovery is effective, but it needs to be more efficient.

Researchers can discover new drugs without understanding how they work. Neuroactive compounds including antipsychotics, antidepressants, and anxiolytics are among the top selling prescription drugs (Alonso et al., 2004; Gu et al., 2010; Alexander et al., 2011; Mojtabai and Olfson, 2014; Olfson et al., 2014). We know some details about how these drugs affect different neurotransmitter signaling pathways. But nobody really knows how these simple molecules change our moods, thoughts, and emotions. Targetbased approaches to central nervous system (CNS) drug discovery have been largely unsuccessful (Paul et al., 2010). However, we can discover new drugs without understanding the details of how they work (Irwin, 1968; Tecott and Nestler, 2004). Historically, many neuroactive drugs were discovered despite totally incorrect therapeutic hypotheses (Sneader, 2005; Kokel and Peterson, 2008; Enna and Williams, 2009). So, although drug discovery and molecular understanding often go hand in hand-it is mostly in that order.

New technologies are changing how researchers use phenotypic assays to discover new drugs. Low throughput assays have limited the field with small sample sizes, narrow scope and limited hypothesis testing. Many key discoveries were made essentially by chance (Sneader, 2005; Enna and Williams, 2009). Now, high throughput assays are enabling a discovery-based approach that relies more on mathematical modeling and massive amounts of data (rather than theory and luck) to identify new drug leads (Schadt et al., 2009). Automated screening platforms do not need mechanistic theories to generate large data sets and identify correlations between compounds and phenotype. As a result, researchers can focus on discovering drugs and drug mechanisms as separate independent endeavors. Here, we review how this data-driven approach to behavioral phenomics is accelerating the pace of neuroactive drug discovery.

\section{HOW MANY NEUROACTIVE DRUGS ARE THERE?}

"How many neuroactive drugs are there?" is a deceptively simple question that can be surprising difficult to answer. Neuroactive drugs are difficult to classify because relationships between compound structure, target and phenotype are often unclear and poorly understood. Structure-based classification is difficult because small structural changes can drastically alter a compound's mechanism of action. Target-based classification is difficult because drug targets are often unknown. Even when in vitro targets are identified, their in vivo relevance is often unclear. One approach is to classify compounds based on behavioral phenotypes or medical utility. But most phenotype-based classifications are subjective and difficult to quantify. How do we know when a drug is an antipsychotic or an antidepressant (Maher et al., 2011)? There are no known molecular causes or biomarker-based diagnostics for most mental disorders (Javitt et al., 2008) and off-label prescriptions are common (Chouinard, 2006; Alexander et al., 2011). So exactly how many neuroactive drugs are there? 
Although the FDA lists thousands of antipsychotics, antidepressants and anxiolytics, most of these compounds fall into just a few structural classes. Consider the antipsychotics. Searching the FDALabel database for "antipsychotic" returns 1,325 hits, but most are mixtures and formulations of identical compounds (U.S. Food and Drug Administration, 2014). The same search in Drugbank returns 42 hits and most are close structural analogs of each other (Kokel and Peterson, 2008). Chemoinformatic algorithms cluster these compounds into a small number of structurally related families (Cao et al., 2008; Backman et al., 2011; Figure 1). Like antipsychotics, the antidepressants and anxiolytics show a similar pattern: There are many individual drugs, but most are structural analogs of a handful of prototypes. These data suggest that many drugs seem to discover themselves, due to the exploitation of prototype molecules (Sneader, 1996).

Most neuroactive drug prototypes were discovered during two broad time periods: pre-history and the mid-1900s. Both waves of discovery coincided with the availability of new chemical compounds alongside relatively widespread human and animal experimentation. The first wave of drugs, discovered in prehistoric times, were found by screening (ingesting) natural products in the environment. Compounds like morphine, alcohol, nicotine, and cocaine were identified based on their strange and unexpected behavioral phenotypes. The second wave of drugs, discovered in the mid-1900s, were found by screening synthetic compounds. These drugs, including the first modern anxiolytics, antipsychotics and antidepressants, were also discovered based on unexpected behavioral phenotypes.

Behavioral phenotyping is an essential part of drug discovery, but it is also the bottleneck (Figure 2). Prototype discovery often starts with the observation of an unexpected behavioral phenotype. Once a prototype has been identified, medicinal chemists generate structural analogs that themselves often have unexpected phenotypes. Researchers use these compounds to test

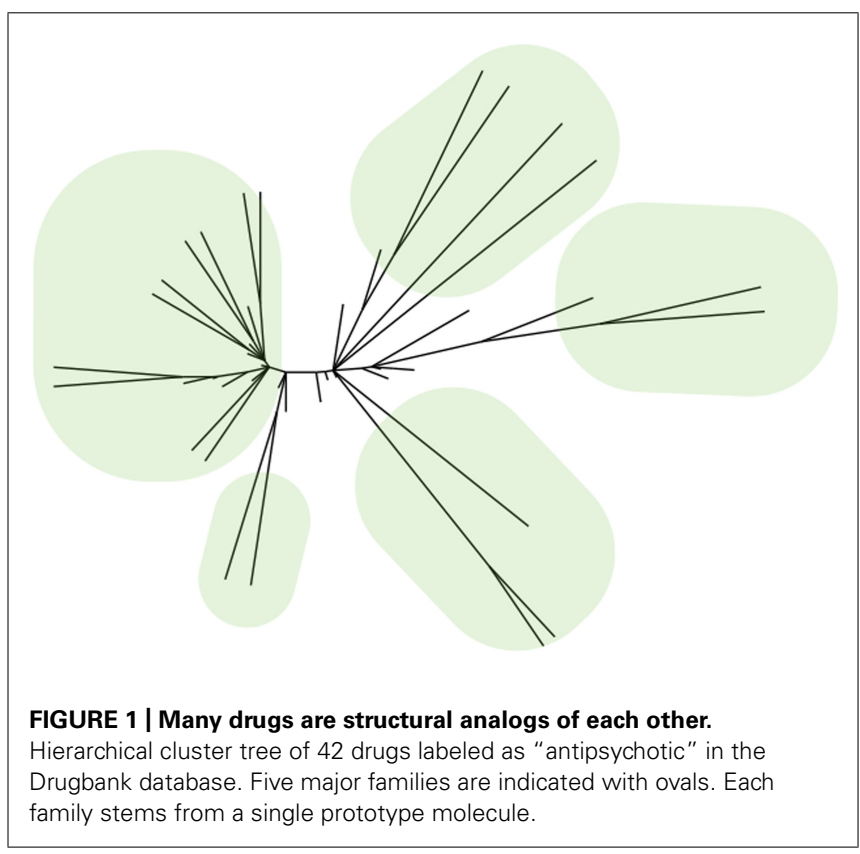

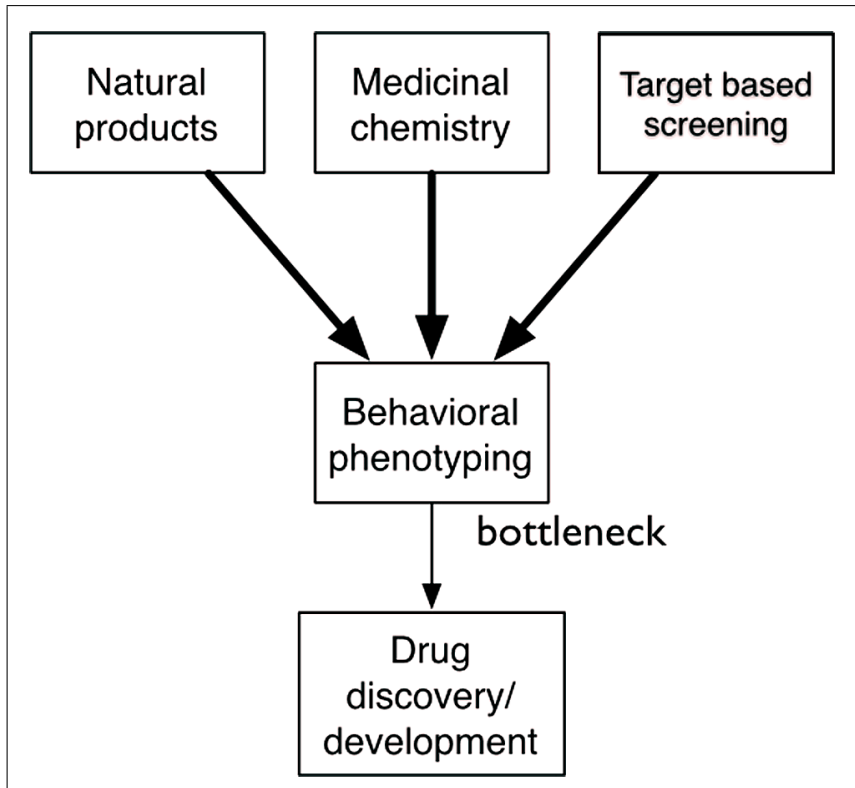

FIGURE 2 | Behavioral phenotyping is a key bottleneck in drug discovery. Although there is an abundance of small molecules from nature, medicinal chemistry, and target-based screening only a very small number are ever tested in behavioral assays, limiting the drug discovery process.

therapeutic hypotheses and search for mechanistic understanding. When molecular targets are identified, researchers search for new ligands that trigger a new round of behavioral phenotyping. In target-based approaches, behavioral phenotyping is deferred until later in the process. Ultimately, the final step of determining efficacy in humans is also a matter of behavioral phenotyping. The process is incredibly effective and has generated most drugs that we use today. Medicinal chemistry can efficiently generate thousands of structural analogs; technologies for in vitro screening are ultra high-throughput. But, decades-old approaches to behavioral phenotyping throttle the drug discovery engine.

\section{WHAT DO ZEBRAFISH DO?}

The zebrafish model system enables researchers to combine complex behavioral phenotyping with high-throughput chemical screening. Like humans, fish are vertebrate animals with complex brains and behaviors. But unlike humans, fish are small enough to fit in 96-well plates and they easily absorb compounds dissolved in the water. These features make zebrafish uniquely well suited for phenotype-based neuroactive drug discovery and enable researchers to scale complex behavioral assays to high-throughput formats.

A frequently asked question about behavior-based drug discovery in zebrafish is "What do zebrafish do?" Personality disorders, depression, and anxiety seem like some of the most complex phenotypes imaginable. Fish do not suffer from these feelings the same way that people do. So, the idea of using fish to discover neuroactive drugs can seem counterintuitive. We tend to think about zebrafish behavior in two different ways: where as some fish behaviors resemble some human behaviors, many others lack obvious human correlates. 
Anthropomorphic assays in fish are very powerful. We immediately empathize with familiar behaviors and their circuitry and mechanisms are likely to be relatively well conserved. For example, researchers use circadian cycles in zebrafish locomotor activity to study mechanism that control sleep behaviors in humans (Prober et al., 2006; Zhdanova, 2006; Yokogawa et al., 2007; Rihel et al., 2010). Researchers also use zebrafish behavior to study pain (Prober et al., 2008), fear (Speedie and Gerlai, 2008; Agetsuma et al., 2010; Mathuru et al., 2012), learned helplessness (Lee et al., 2010), feeding (Gahtan et al., 2005; Del Bene et al., 2010; Bianco et al., 2011), courtship (Darrow and Harris, 2004), learning (Valente et al., 2012), vision (Emran et al., 2007), hearing (Gleason et al., 2009), touch (Low et al., 2010, 2011, 2012), social interactions (Pérez-Escudero and de Polavieja, 2011; Mahabir et al., 2013; Qin et al., 2014), anxiety (Stewart et al., 2012), and decision making (Arganda et al., 2012).

By contrast, it is more difficult to empathize with fishspecific phenotypes. The practical implications of understanding fundamental fish behaviors are not always obvious. And it is easy focus on their differences rather then their similarities. Nevertheless, neuroactive drugs affect fish behavior in specific and reproducible ways via conserved molecular mechanisms. Adult zebrafish, differentially change their swimming and three-dimensional tank diving behaviors in response to many neuroactive compounds (Cachat etal., 2011; Grossman et al., 2011; Stewart etal., 2011; Kyzar etal., 2012a, 2013; Williams etal., 2012; Robinson etal., 2013; Stewart and Kalueff, 2014). In larvae, fish specific behaviors like spontaneous swimming (Wyart et al., 2009), the optokinetic reflex (Emran et al., 2007) and photomotor response (Kokel etal., 2013b) can be used to understand neuronal signaling, rapidly identify novel neuroactive compounds and predict their mechanisms of action (Kokel et al., 2010; Rihel et al., 2010). The key challenge is learning how to decode complex patterns of behavior to understand which pathways are being modulated—and how they may affect human health.

\section{A DISCOVERY-BASED APPROACH}

Some of the most exciting developments in behavioral phenomics are coming from two very different models: humans and zebrafish. Compared to other animals, human behaviors are probably the most complex, variable and challenging to measure. So it is somewhat surprising that human behavioral phenomics is advancing so rapidly. One reason is that substantial investments by internet technology companies have increased the scale of digital record keeping and chemobehavioral phenotyping. Large medical databases link people's genotype, phenotype and prescription drug records. Researchers are mining these databases to identify unanticipated drug side effects and repurpose drugs for new indications (Dudley et al., 2010).

Human behavioral phenomics is a powerful way to approach drug repurposing, but it cannot be used for chemical screening. Governmental and institutional regulations limit large-scale human studies to compounds that are already approved by the FDA (thankfully). Researchers will need other model organisms, like zebrafish, to systematically discover new molecular entities. Until recently, tools for high throughput behavioral phenotyping were unavailable. But new technologies are changing the drug discovery landscape.

\section{AUTOMATED SOLUTIONS}

Automated technologies are making behavior-based chemical screening in zebrafish a more effective, efficient and systematic way to discover neuroactive compounds. Three aspects of automation are changing the field of behavior-based drug screening: robotics, analytics, and academic industrial collaboration. These changes are a small part of larger global trends in computing technology. As sophisticated processors, programming languages, and rapid prototyping tools become more accessible, individual scientists and small academic laboratories are innovating alongside larger biotechnology and pharmaceutical companies.

Robotic solutions are growing to meet nearly every early step of the screening process including fish breeding, sorting, and phenotyping. Robotic aquaculture racks automate feeding cycles and monitor water quality. Specialized breeding tanks produce thousands of synchronized embryos (Adatto etal., 2011). Flow cytometry platforms sort zebrafish into 96-well plates. And imaging platforms automate morphological and behavioral phenotyping (Burgess and Granato, 2007a; Pardo-Martin et al., 2010a, 2013; Ahrens et al., 2012; Engert, 2012; Wittmann et al., 2012). For example, researchers have developed an elegant and powerful (freely available) software package, FLOTE, for automated tracking of precise kinematic events in larval zebrafish (Burgess and Granato, 2007a). The software has already been used to analyze startle modulation, light adaptation, and navigation (Burgess and Granato, 2007a,b; Burgess et al., 2009, 2010; Jain et al., 2011; Fernandes et al., 2012). The software has also been used to find compounds that modulate memory formation in larval zebrafish (Wolman et al., 2011). Although not yet used for drug screening, recent advances in whole-brain functional imaging record patterns of firing activity of individual cells in large populations of neurons (Ahrens et al., 2012, 2013; Kokel et al., 2013b; Muto et al., 2013; Satou et al., 2013; Portugues et al., 2014) and will likely add massive amounts data to the behavioral pharmacology field. As behavioral datasets grow, researchers are applying new analytical approaches to explore, organize, and discover correlations between phenotypic patterns and compound treatments.

Academic-industrial partnerships are improving zebrafish phenotyping and phenotype-based approaches to drug discovery. The innovations flow both ways, from academia to industry and industry to academia. Acquifer (http://www.acquifer.de), a new biotech company with roots in academic automated zebrafish phenotyping, is developing network platforms for managing huge amounts of data from zebrafish phenotypic screens. Commercial imaging platforms, like the Vertebrate Automated Screening Technology marketed is based on academic innovations (Pardo-Martin et al., 2010b, 2013; Chang et al., 2012). When equipment is too expensive, academic bioinstrumentation laboratories are working to develop more affordable do-it-yourself kits (Alper, 2009; Marzullo and Gage, 2012). As sophisticated rapid prototyping tools become more accessible (like 3D printers, open source programming languages, and cheap microcontrollers) the pace of innovation is accelerating. 


\section{SCALING BEHAVIORAL DATABASES INTO CONNECTIVITY MAPS}

Today, database-linked tools for analyzing gene expression data and behavioral data look very different. Behavioral databases tend to be designed for finding and summarizing data via search field descriptors like compound name, genes name and strain name. For example, the Zebrafish Neurophenome Database (ZND) is a publically available database designed to provide a comprehensive resource of neurobehavioral phenotypes in adult zebrafish (Green et al., 2012; Kyzar et al., 2012b; Kalueff et al., 2013). To search the ZND, a researcher uses drop-down fields to select investigator and drugs of interest to experimental results and drug effects that are often presented as textual descriptions. Similarly, large-scale mouse phenotyping projects like the Mouse Phenome Database (MPD) at The Jackson Laboratory allow users to find, visualize and analyze mouse behavioral phenotypes across different strains and conditions (Maddatu et al., 2012). The MPD stores a large amount of standardized, quantifiable and comparable data (like weight and grip strength). The MPD also provides a variety of tools to analyze results (including tools to find strains that best fit phenotypic criteria). But, as phenotypic databases grow ever larger, they will enable more complex data-driven queries.

Given sufficiently rich behavioral phenotyping, it should be possible to build a connectivity map to systematically identify neuroactive compounds and sort them into phenotypic classes. For example, the Connectivity Map is designed to use gene expression data as a discovery framework by allowing researchers to use gene expression signatures to query the data for closely related perturbagens (Lamb et al., 2006; Lamb, 2007). As a result, one can use the data itself to identify correlations, perform cluster analyses and identify outliers. Analyses that were originally developed for applications like speech recognition and social networking can just as easily be applied to analyzing zebrafish phenotypes. And these analyses allow new questions about large diverse data sets. We imagine that someday soon, it may be possible to query large behavioral databases with BLAST-like and speech recognition tools. This could allow researchers to identify all compounds with similar behavioral phenotypes, link genetic mutants to small molecule treatments and identify new treatments with totally novel phenotypes. Will it be possible to identify just the right pattern of fish behaviors to accurately identify drugs with complex activities in humans (like antipsychotics and antidepressants)? Future studies may provide the answer.

\section{WHAT ARE WE LIKELY TO FIND?}

Given that so few compounds have been tested in animals, large-scale behavioral screens are almost guaranteed to identify new neuroactive compounds. These studies will provide highresolution maps of how small molecules affect the brain and behavior. But what kinds of compounds are likely to be discovered? Are we really likely to identify new compounds with new mechanisms of action? Or just more of the same kinds of drugs we already have? The data supports both arguments.

On the one hand, one could argue that behavior-based drug screening has been saturated: Multiple classes of antipsychotics, antidepressants, and anxiolytics have already been identified. One possibility is that the low throughput non-systematic approaches employed in the past have already identified all the neuroactive drugs worth discovering. Alternatively, it is interesting to speculate that compounds with antipsychotic, antidepressant and anxiolytic effects may be relatively common. If so, large-scale screens would likely identify a variety of new psychotropic drug prototypes with a range of phenotypic and mechanistic profiles, including totally new structures, mechanisms, and phenotypes.

Large zebrafish behavior-based chemical screens are already identifying a variety of new compounds. Some of first neuroactive compounds to be discovered in zebrafish, str1, and str2, were novel acetylcholinesterase inhibitors (Kokel et al., 2010). These compounds were new molecular entities, but they were not first in class compounds. These compounds may provide modest advantages over current treatment options. But identifying novel structures with novel targets and mechanisms would have a greater impact. One potential way to identify compounds with novel mechanisms is to identify compounds that cause outlier phenotypes in behavioral databases. If one compound in ten thousand causes a unique behavioral phenotype, this suggests it may be working through a new (and rare) mechanism of action. For example, a new kind of light controllable rapidly reversible TrpA1 ligand, optovin, was recently discovered in just this way (Kokel et al., 2013a). Several novel light activated molecules have been developed using zebrafish behavioral readouts (Szobota et al., 2007; Janovjak et al., 2010; Levitz et al., 2013). This suggests that truly novel compounds are waiting to be found, if only we use the right methods to look for them.

\section{MODIFIER SCREENS: CHEMICAL AND GENETIC MODELS}

Although wild-type phenotypes may be useful for identifying certain compounds, we can also use chemical and genetic tools to model specific disease states. These disease models combine the advantages of unbiased phenotypic screening with readouts that are specifically designed to target certain kinds of compounds. In one recent example, researchers identified a zebrafish mutant (in the Scnla gene) and then used this model to screen for potential treatments for Dravet syndrome (caused by mutations in the homologous human gene; Baraban et al., 2013). These researchers identified an FDA approved compound that suppressed the fish phenotype, suggesting that the approach may be a powerful way to identify therapeutics for this specific disorder. This work elegantly illustrates the potential for genetic models in zebrafish to identify desperately needed targeted therapeutics with potential utility in humans. One can imagine many variations on this theme. CRISPCas technology is revolutionizing zebrafish researchers' ability to efficiently generate knockout and knock-in models (Hwang et al., 2013a,b; Auer et al., 2014). Transgenic overexpression models phenocopy aspects of neurodegenerative and other dominant diseases (Bai et al., 2007; Olson etal., 2010). And, due to the ease of chemical manipulations, researchers have run large-scale modifier screens in chemically treated disease models (Baraban et al., 2005).

\section{WHOLE ORGANISM PHENOTYPING: BLOOD-BRAIN BARRIER AND TOXICOLOGY}

Researchers can expand phenotypic readouts to encompass almost any aspect the organism including blood-brain barrier (BBB), 
toxicity, and cardiovascular readouts. Larval zebrafish develop a functional $\mathrm{BBB}$ with size exclusion and transport pumps including those that are similar to mammals (Jeong et al., 2008). So researchers have some reason to believe compounds with CNS activity in fish may also penetrate the BBB in mammals. Similarly, potentially toxic compounds can be screened for unwanted and unexpected toxic or cardiovascular side effects. One could potentially capture data on zebrafish development, behavior and heart rate simultaneously in a high-throughput and automated fashion. Because researchers can apply diverse phenotyping assays, zebrafish are an exciting model for toxicology in addition to drug discovery.

\section{THE CHALLENGE OF TRANSLATING FROM FISH TO HUMANS}

Despite the power of new technologies, there are substantial fundamental challenges to translating CNS drug discovery from fish to humans. Most investigational new drugs fail when they are finally tested (for efficacy) in humans (Paul et al., 2010). There are many reasons why preclinical predictions from any model system would fail to translate, but lack of rigor should not be one of them.

Inefficient animal studies contribute to publication bias, decrease scientific rigor, and limit the drug discovery process. Compared to zebrafish, studies in larger animals, like mice, are relatively expensive and require substantial amounts of test compounds. Due to these costs, some large-animal studies tend to be underpowered, which contributes to irreproducible results (Landis et al., 2012). Zebrafish enable a level of rigor and reproducibility that can be difficult to achieve in larger model organisms, simply because the assays can be easily reproduced on larger scales. Hypotheses can be tested on thousands of animals, rather than just a handful, at small cost in time and other resources. For example, treating a single mouse (at $10 \mathrm{mg} / \mathrm{kg}$ ) requires approximately $100 \mathrm{X}$ more compound than is needed to treat a well of zebrafish (at $10 \mu \mathrm{M}$ ). When researchers increase sample size it becomes easier to find true signals amongst the noise. However, even if new compounds can be discovered with reproducible effects on zebrafish behavior, substantial challenges remain to translate these discoveries for improving human health.

Many compounds work in humans, many work in zebrafish, and some fraction is likely to work in both-although the exact level of overlap is difficult to predict (Figure 3). Humans and zebrafish are closely related (Howe et al., 2013), but there are many differences at the phenotypic, neuronal network, and molecular levels. When a new bioactive compound is first discovered in zebrafish, it will be difficult to predict its potential therapeutic utility in humans. Many compounds that appear to work well in mice and other animal models subsequently fail to translate to humans. The same will surely be true of zebrafish. The problem is especially relevant in neuropharmacology, where CNS disorders are poorly understood and difficult to model. Despite the challenges, in the upcoming years we are likely to see at least a few compounds identified in zebrafish screens translate from bench to bedside.

\section{ACKNOWLEDGMENTS}

The authors would like to acknowledge Michael Keiser and John Irwin for chemoinformatic advice. Also, Sam Enna for publically discussing "targephilia" (obsession with, and excessive focus on,

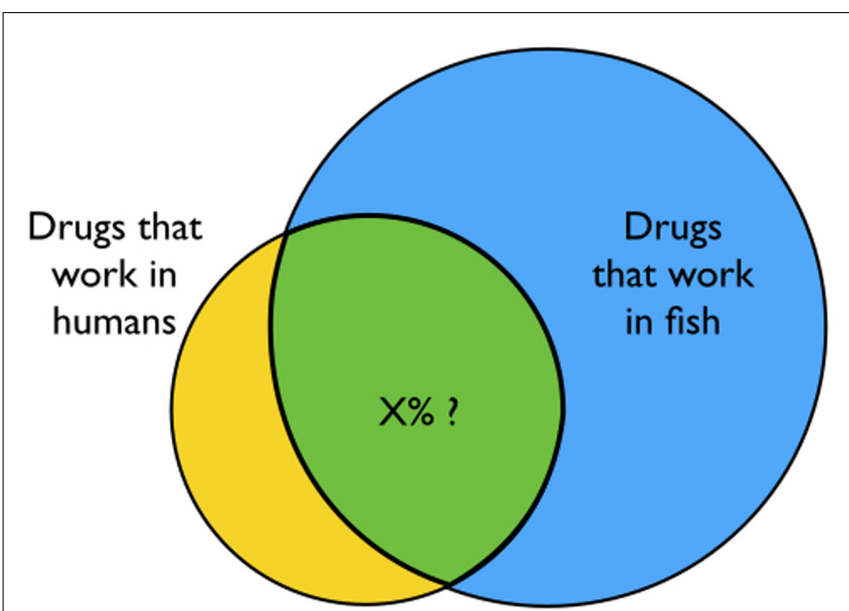

FIGURE 3 | How many drugs work in both humans and zebrafish? Not all drugs that work in zebrafish will also work in humans. Because so many more compounds can be screened in zebrafish, it is likely that some will translate to the clinic. However, the precise level of overlap (indicated by $\mathrm{X}$ ) is difficult to predict.

sites of drug action) and the "Paleo era" of drug discovery. Many of the ideas and concepts presented here developed from discussions with Randy Peterson. We express our gratitude for their input and support.

\section{REFERENCES}

Adatto, I., Lawrence, C., Thompson, M., and Zon, L. I. (2011). A new system for the rapid collection of large numbers of developmentally staged zebrafish embryos. PLoS ONE 6:e21715. doi: 10.1371/journal.pone.0021715

Agetsuma, M., Aizawa, H., Aoki, T., Nakayama, R., Takahoko, M., Goto, M., et al. (2010). The habenula is crucial for experience-dependent modification of fear responses in zebrafish. Nat. Neurosci. 13, 1354-1356. doi: 10.1038/nn.2654

Ahrens, M. B., Li, J. M., Orger, M. B., Robson, D. N., Schier, A. F., Engert, F., et al. (2012). Brain-wide neuronal dynamics during motor adaptation in zebrafish. Nature 485, 471-477. doi: 10.1038/nature11057

Ahrens, M. B., Orger, M. B., Robson, D. N., Li, J. M., and Keller, P. J. (2013). Wholebrain functional imaging at cellular resolution using light-sheet microscopy. Nat. Methods 10, 413-420. doi: 10.1038/nmeth.2434

Alexander, G. C., Gallagher, S. A., Mascola, A., Moloney, R. M., and Stafford, R. S. (2011). Increasing off-label use of antipsychotic medications in the United States, 1995-2008. Pharmacoepidemiol. Drug Saf. 20, 177-184. doi: 10.1002/pds.2082

Alonso, J., Angermeyer, M. C., Bernert, S., Bruffaerts, R., Brugha, T. S., Bryson, H., et al. (2004). Psychotropic drug utilization in Europe: results from the European Study of the Epidemiology of Mental Disorders (ESEMeD) project. Acta Psychiatr. Scand. Suppl. 109, 55-64. doi: 10.1111/j.1600-0047.2004.00325.x

Alper, J. (2009). Biotech in the basement. Nat. Biotechnol. 27, 1077-1078. doi: $10.1038 /$ nbt1209-1077

Arganda, S., Pérez-Escudero, A., and de Polavieja, G. G. (2012). A common rule for decision making in animal collectives across species. Proc. Natl. Acad. Sci. U.S.A. 109, 20508-20513. doi: 10.1073/pnas.1210664109

Auer, T. O., Duroure, K., De Cian, A., Concordet, J.-P., and Del Bene, F. (2014). Highly efficient CRISPR/Cas9-mediated knock-in in zebrafish by homologyindependent DNA repair. Genome Res. 24, 142-153. doi: 10.1101/gr.161638.113

Backman, T. W. H., Cao, Y., and Girke, T. (2011). ChemMine tools: an online service for analyzing and clustering small molecules. Nucleic Acids Res. 39, W486-W491. doi: 10.1093/nar/gkr320

Bai, Q., Garver, J. A., Hukriede, N. A., and Burton, E. A. (2007). Generation of a transgenic zebrafish model of Tauopathy using a novel promoter element derived from the zebrafish eno2 gene. Nucleic Acids Res. 35, 6501-6516. doi: 10.1093/nar/gkm608 
Baraban, S. C., Dinday, M. T., and Hortopan, G. A. (2013). Drug screening in Scn1a zebrafish mutant identifies clemizole as a potential Dravet syndrome treatment. Nat. Commun. 4, 2410. doi: 10.1038/ncomms3410

Baraban, S. C., Taylor, M. R., Castro, P. A., and Baier, H. (2005). Pentylenetetrazole induced changes in zebrafish behavior, neural activity and c-fos expression. Neuroscience 131, 759-768. doi: 10.1016/j.neuroscience.2004.11.031

Bianco, I. H., Kampff, A. R., and Engert, F. (2011). Prey capture behavior evoked by simple visual stimuli in larval zebrafish. Front. Syst. Neurosci. 5:101. doi: 10.3389/fnsys.2011.00101

Burgess, H. A., and Granato, M. (2007a). Modulation of locomotor activity in larval zebrafish during light adaptation. J. Exp. Biol. 210, 2526-2539. doi: 10.1242/jeb.003939

Burgess, H. A., and Granato, M. (2007b). Sensorimotor gating in larval zebrafish. J. Neurosci. 27, 4984-4994. doi: 10.1523/JNEUROSCI.0615-07.2007

Burgess, H. A., Johnson, S. L., and Granato, M. (2009). Unidirectional startle responses and disrupted left-right co-ordination of motor behaviors in robo3 mutant zebrafish. Genes Brain Behav. 8, 500-511. doi: 10.1111/j.1601183X.2009.00499.x

Burgess, H. A., Schoch, H., and Granato, M. (2010). Distinct retinal pathways drive spatial orientation behaviors in zebrafish navigation. Curr. Biol. 20, 381-386. doi: 10.1016/j.cub.2010.01.022

Cachat, J., Stewart, A., Utterback, E., Hart, P., Gaikwad, S., Wong, K., et al. (2011). Three-dimensional neurophenotyping of adult zebrafish behavior. PLoS ONE 6:e17597. doi: 10.1371/journal.pone.0017597

Cao, Y., Charisi, A., Cheng, L.-C., Jiang, T., and Girke, T. (2008). ChemmineR: a compound mining framework for R. Bioinformatics 24, 1733-1734. doi 10.1093/bioinformatics/btn307

Chang, T.-Y., Pardo-Martin, C., Allalou, A., Wählby, C., and Yanik, M. F. (2012). Fully automated cellular-resolution vertebrate screening platform with parallel animal processing. Lab Chip 12, 711-716. doi: 10.1039/c1lc20849g

Chouinard, G. (2006). The search for new off-label indications for antidepressant, antianxiety, antipsychotic and anticonvulsant drugs. J. Psychiatry Neurosci. 31, $168-176$.

Darrow, K. O., and Harris, W. A. (2004). Characterization and development of courtship in zebrafish, Danio rerio. Zebrafish 1, 40-45. doi $10.1089 / 154585404774101662$

Del Bene, F., Wyart, C., Robles, E., Tran, A., Looger, L., Scott, E. K., et al. (2010). Filtering of visual information in the tectum by an identified neural circuit. Science 330, 669-673. doi: 10.1126/science.1192949

Dudley, J. T., Schadt, E., Sirota, M., Butte, A. J., and Ashley, E. (2010). Drug discovery in a multidimensional world: systems, patterns, and networks. J. Cardiovasc. Transl. Res. 3, 438-447. doi: 10.1007/s12265-010-9214-6

Emran, F., Rihel, J., Adolph, A. R., Wong, K. Y., Kraves, S., and Dowling, J. E. (2007). OFF ganglion cells cannot drive the optokinetic reflex in zebrafish. Proc. Natl. Acad. Sci. U.S.A. 104, 19126-19131. doi: 10.1073/pnas.0709337104

Engert, F. (2012). Fish in the matrix: motor learning in a virtual world. Front. Neural Circuits 6:125. doi: 10.3389/fncir.2012.00125

Enna, S. J., and Williams, M. (2009). Challenges in the search for drugs to treat central nervous system disorders. J. Pharmacol. Exp. Ther. 329, 404-411. doi: 10.1124/jpet.108.143420

Fernandes, A. M., Fero, K., Arrenberg, A. B., Bergeron, S. A., Driever, W., and Burgess, H. A. (2012). Deep brain photoreceptors control light-seeking behavior in zebrafish larvae. Curr. Biol. 22, 2042-2047. doi: 10.1016/j.cub.2012.08.016

Gahtan, E., Tanger, P., and Baier, H. (2005). Visual prey capture in larval zebrafish is controlled by identified reticulospinal neurons downstream of the tectum. J. Neurosci. 25, 9294-9303. doi: 10.1523/JNEUROSCI.2678-05.2005

Gleason, M. R., Nagiela, A., Jametb, S., Vologodskaiaa, M., López-Schiera, H., and Hudspetha, A. J. (2009). The transmembrane inner ear (Tmie) protein is essential for normal hearing and balance in the zebrafish. Proc. Natl. Acad. Sci. U.S.A. 106, 21347-21352. doi: 10.1073/pnas.0911632106

Green, J., Collins, C., Kyzar, E. J., Pham, M., Roth, A., Gaikwad, S., et al. (2012). Automated high-throughput neurophenotyping of zebrafish social behavior. I. Neurosci. Methods 210, 266-271. doi: 10.1016/j.jneumeth.2012.07.017

Grossman, L., Stewart, A., Gaikwad, S., Utterback, E., Wu, N., Dileo, J., et al. (2011). Effects of piracetam on behavior and memory in adult zebrafish. Brain Res. Bull. 85, 58-63. doi: 10.1016/j.brainresbull.2011.02.008

Gu, Q., Dillon, C. F., and Burt, V. L. (2010). Prescription drug use continues to increase: U.S. prescription drug data for 2007-2008. NCHS Data Brief 42, 1-8.
Howe, K., Clark, M. D., Torroja, C. F., Torrance, J., Berthelot, C., Muffato, M., et al. (2013). The zebrafish reference genome sequence and its relationship to the human genome. Nature 496, 498-503. doi: 10.1038/nature12111

Hwang, W. Y., Fu, Y., Reyon, D., Maeder, M. L., Kaini, P., Sander, J. D., et al. (2013a). Heritable and precise zebrafish genome editing using a CRISPR-Cas system. PLoS ONE 8:e68708. doi: 10.1371/journal.pone.0068708

Hwang, W. Y., Fu, Y., Reyon, D., Maeder, M. L., Tsai, S. Q., Sander, J. D., et al. (2013b). Efficient genome editing in zebrafish using a CRISPR-Cas system. Nat. Biotechnol. 31, 227-229. doi: 10.1038/nbt.2501

Irwin, S. (1968). Comprehensive observational assessment: Ia. A systematic, quantitative procedure for assessing the behavioral and physiologic state of the mouse. Psychopharmacology (Berl.) 13, 222-257. doi: 10.1007/BF00401402

Jain, R. A., Wolman, M. A., Schmidt, L. A., Burgess, H. A., and Granato, M. (2011). Molecular-genetic mapping of zebrafish mutants with variable phenotypic penetrance. PLOS ONE 6:e26510. doi: 10.1371/journal.pone. 0026510

Janovjak, H., Szobota, S., Wyart, C., Trauner, D., and Isacoff, E. Y. (2010). A light-gated, potassium-selective glutamate receptor for the optical inhibition of neuronal firing. Nat. Neurosci. 13, 1027-1032. doi: 10.1038/nn.2589

Javitt, D. C., Spencer, K. M., Thaker, G. K., Winterer, G., and Hajós, M. (2008). Neurophysiological biomarkers for drug development in schizophrenia. Nat. Rev. Drug Discov. 7, 68-83. doi: 10.1038/nrd2463

Jeong, J.-Y., Kwon, H. B., Ahn, J. C., Kang, D., Kwon, S. H., Park, J. A., et al. (2008). Functional and developmental analysis of the blood-brain barrier in zebrafish. Brain Res. Bull. 75, 619-628. doi: 10.1016/j.brainresbull.2007.10.043

Kalueff, A. V., Gebhardt, M., Stewart, A. M., Cachat, J. M., Brimmer, M., Chawla, J. S., et al. (2013). Towards a comprehensive catalog of zebrafish behavior 1.0 and beyond. Zebrafish 10, 70-86. doi: 10.1089/zeb.2012.0861

Kokel, D., Bryan, J., Laggner, C., White, R., Cheung, C. Y., Mateus, R., et al. (2010). Rapid behavior-based identification of neuroactive small molecules in the zebrafish. Nat. Chem. Biol. 6, 231-237. doi: 10.1038/nchembio.307

Kokel, D., Cheung, C. Y., Mills, R., Coutinho-Budd, J., Huang, L., Setola, V., et al. (2013a). Photochemical activation of TRPAl channels in neurons and animals. Nat. Chem. Biol. 9, 257-263. doi: 10.1038/nchembio.1183

Kokel, D., Dunn, T. W., Ahrens, M. B., Alshut, R., Cheung, C. Y., Saint-Amant, L., et al. (2013b). Identification of nonvisual photomotor response cells in the vertebrate hindbrain. J. Neurosci. 33, 3834-3843. doi: 10.1523/JNEUROSCI.368912.2013

Kokel, D., and Peterson, R. T. (2008). Chemobehavioural phenomics and behaviourbased psychiatric drug discovery in the zebrafish. Brief. Funct. Genomic. Proteomic. 7, 483-490. doi: 10.1093/bfgp/eln040

Kyzar, E. J., Collins, C., Gaikwad, S., Green, J., Roth, A., Monnig, L., et al. (2012a). Effects of hallucinogenic agents mescaline and phencyclidine on zebrafish behavior and physiology. Prog. Neuropsychopharmacol. Biol. Psychiatry 37, 194-202. doi: 10.1016/j.pnpbp.2012.01.003

Kyzar, E., Zapolsky, I., Green, J., Gaikwad, S., Pham, M., Collins, C., et al. (2012b). The Zebrafish Neurophenome Database (ZND): a dynamic open-access resource for zebrafish neurophenotypic data. Zebrafish 9, 8-14. doi: 10.1089/zeb.2011. 0725

Kyzar, E., Stewart, A. M., Landsman, S., Collins, C., Gebhardt, M., Robinson, K., et al. (2013). Behavioral effects of bidirectional modulators of brain monoamines reserpine and d-amphetamine in zebrafish. Brain Res. 1527, 108-116. doi: 10.1016/j.brainres.2013.06.033

Lamb, J. (2007). The Connectivity Map: a new tool for biomedical research. Nat. Rev. Cancer 7, 54-60. doi: 10.1038/nrc2044

Lamb, J., Crawford, E. D., Peck, D., Modell, J. W., Blat, I. C., Wrobel, M. J., et al. (2006). The Connectivity Map: using gene-expression signatures to connect small molecules, genes, and disease. Science 313, 1929-1935. doi: $10.1126 /$ science. 1132939

Landis, S. C., Amara, S. G., Asadullah, K., Austin, C. P., Blumenstein, R., Bradley, E. W., et al. (2012). A call for transparent reporting to optimize the predictive value of preclinical research. Nature 490, 187-191. doi: 10.1038/nature11556

Lee, A., Mathuru, A. S., Teh, C., Kibat, C., Korzh, V., Penney, T. B., et al. (2010). The habenula prevents helpless behavior in larval zebrafish. Curr. Biol. 20, 2211-2216. doi: 10.1016/j.cub.2010.11.025

Levitz, J., Pantoja, C., Gaub, B., Janovjak, H., Reiner, A., Hoagland, A., et al. (2013). Optical control of metabotropic glutamate receptors. Nat. Neurosci. 16, 507-516. doi: $10.1038 /$ nn.3346 
Low, S. E., Amburgey, K., Horstick, E., Linsley, J., Sprague, S. M., Cui, W. W., et al. (2011). TRPM7 is required within zebrafish sensory neurons for the activation of touch-evoked escape behaviors. J. Neurosci. 31, 11633-11644. doi: 10.1523/JNEUROSCI.4950-10.2011

Low, S. E., Ryan, J., Sprague, S. M., Hirata, H., Cui, W. W., Zhou, W., et al. (2010). touché Is required for touch-evoked generator potentials within vertebrate sensory neurons. J. Neurosci. 30, 9359-9367. doi: 10.1523/JNEUROSCI.1639-10.2010

Low, S. E., Woods, I. G., Lachance, M., Ryan, J., Schier, A. F., and Saint-Amant, L. (2012). Touch responsiveness in zebrafish requires voltage-gated calcium channel 2.1b. J. Neurophysiol. 108, 148-159. doi: 10.1152/jn.00839.2011

Maddatu, T. P., Grubb, S. C., Bult, C. J., and Bogue, M. A. (2012). Mouse Phenome Database (MPD). Nucleic Acids Res. 40, D887-D894. doi: 10.1093/nar/gkr1061

Mahabir, S., Chatterjee, D., Buske, C., and Gerlai, R. (2013). Maturation of shoaling in two zebrafish strains: a behavioral and neurochemical analysis. Behav. Brain Res. 247, 1-8. doi: 10.1016/j.bbr.2013.03.013

Maher, A. R., Maglione, M., Bagley, S., Suttorp, M., Hu, J. H., Ewing, B., et al. (2011). Efficacy and comparative effectiveness of atypical antipsychotic medications for off-label uses in adults: a systematic review and meta-analysis. JAMA 306, 1359 1369. doi: 10.1001/jama.2011.1360

Marzullo, T. C., and Gage, G. J. (2012). The SpikerBox: a low cost, open-source bioamplifier for increasing public participation in neuroscience inquiry. PLoS ONE 7:e30837. doi: 10.1371/journal.pone.0030837

Mathuru, A. S., Kibat, C., Cheong, W. F., Shui, G., Wenk, M. R., Friedrich, R. W., et al. (2012). Chondroitin fragments are odorants that trigger fear behavior in fish. Curr. Biol. 22, 538-544. doi: 10.1016/j.cub.2012.01.061

Mojtabai, R., and Olfson, M. (2014). National trends in long-term use of antidepressant medications: results from the U.S. National Health and Nutrition Examination Survey. J. Clin. Psychiatry 75, 169-177. doi: 10.4088/JCP.13m08443

Muto, A., Ohkura, M., Abe, G., Nakai, J., and Kawakami, K. (2013). Real-time visualization of neuronal activity during perception. Curr. Biol. 23, 307-311. doi: 10.1016/j.cub.2012.12.040

Olfson, M., Kroenke, K., Wang, S., and Blanco, C. (2014). Trends in office-based mental health care provided by psychiatrists and primary care physicians. J. Clin. Psychiatry 75, 247-253. doi: 10.4088/JCP.13m08834

Olson, B. D., Sgourdou, P., and Downes, G. B. (2010). Analysis of a zebrafish behavioral mutant reveals a dominant mutation in atp2a1/SERCA1. Genesis 48, 354-361. doi: 10.1002/dvg.20631

Pardo-Martin, C., Allalou, A., Medina, J., Eimon, P. M., Wählby, C., and Fatih Yanik, M. (2013). High-throughput hyperdimensional vertebrate phenotyping. Nat. Commun. 4, 1467. doi: 10.1038/ncomms 2475

Pardo-Martin, C., Chang, T. Y., Koo, B. K., Gilleland, C. L., Wasserman, S. C., and Yanik, M. F. (2010a). High-throughput in vivo vertebrate screening. Nat. Methods 7, 634-636. doi: 10.1038/nmeth.1481

Pardo-Martin, C., Chang, T. Y., Koo, B. K., Gilleland, C. L., Wasserman, S. C., and Yanik, M. F. (2010b). High-throughput in vivo vertebrate screening. Nat Methods 7, 634-636. doi: 10.1038/nmeth.1481

Paul, S. M., Mytelka, D. S., Dunwiddie, C. T., Persinger, C. C., Munos, B. H., Lindborg, S. R., et al. (2010). How to improve R\&D productivity: the pharmaceutical industry's grand challenge. Nat. Rev. Drug Discov. 9, 203-214.

Pérez-Escudero, A., and de Polavieja, G. G. (2011). Collective animal behavior from Bayesian estimation and probability matching. PLoS Comput. Biol. 7:e1002282. doi: 10.1371/journal.pcbi.1002282

Portugues, R., Feierstein, C. E., Engert, F., and Orger, M. B. (2014). Whole-brain activity maps reveal stereotyped, distributed networks for visuomotor behavior. Neuron 81, 1328-1343. doi: 10.1016/j.neuron.2014.01.019

Prober, D. A., Rihel, J., Onah, A. A., Sung, R. J., and Schier, A. F. (2006). Hypocretin/orexin overexpression induces an insomnia-like phenotype in zebrafish. $J$. Neurosci. 26, 13400-13410. doi: 10.1523/JNEUROSCI.4332-06.2006

Prober, D. A., Zimmerman, S., Myers, B. R., McDermott, B. M. Jr., Kim, S. H., Caron, S., et al. (2008). Zebrafish TRPA1 channels are required for chemosensation but not for thermosensation or mechanosensory hair cell function. J. Neurosci. 28, 10102-10110. doi: 10.1523/JNEUROSCI.2740-08.2008

Qin, M., Wong, A., Seguin, D., and Gerlai, R. (2014). Induction of social behavior in zebrafish: live versus computer animated fish as stimuli. Zebrafish 11, 185-197. doi: 10.1089/zeb.2013.0969

Rihel, J., Prober, D. A., Arvanites, A., Lam, K., Zimmerman, S., Jang, S., et al. (2010). Zebrafish behavioral profiling links drugs to biological targets and rest/wake regulation. Science 327, 348-351. doi: 10.1126/science.1183090
Robinson, K. S., Stewart, A. M., Cachat, J., Landsman, S., Gebhardt, M., and Kalueff, A. V. (2013). Psychopharmacological effects of acute exposure to kynurenic acid (KYNA) in zebrafish. Pharmacol. Biochem. Behav. 108, 54-60. doi: 10.1016/j.pbb.2013.04.002

Satou, C., Kimura, Y., Hirata, H., Suster, M. L., Kawakami, K., and Higashijima, S. (2013). Transgenic tools to characterize neuronal properties of discrete populations of zebrafish neurons. Development 140, 3927-3931. doi: 10.1242/dev.099531

Schadt, E. E., Friend, S. H., and Shaywitz, D. A. (2009). A network view of disease and compound screening. Nat. Rev. Drug Discov. 8, 286-295. doi: 10.1038/nrd2826

Sneader, W. (1996). Drug Prototypes and Their Exploitation. Hoboken: Wiley.

Sneader, W. (2005). Drug Discovery. Hoboken: John Wiley \& Sons. doi: $10.1002 / 0470015535$

Speedie, N., and Gerlai, R. (2008). Alarm substance induced behavioral responses in zebrafish (Danio rerio). Behav. Brain Res. 188, 168-177. doi: 10.1016/j.bbr.2007.10.031

Stewart, A., Gaikwad, S., Kyzar, E., Green, J., Roth, A., and Kalueff, A. V. (2012). Modeling anxiety using adult zebrafish: a conceptual review. Neuropharmacology 62, 135-143. doi: 10.1016/j.neuropharm.2011.07.037

Stewart, A., Riehl, R., Wong, K., Green, J., Cosgrove, J., Vollmer, K., et al. (2011). Behavioral effects of MDMA ('ecstasy') on adult zebrafish. Behav. Pharmacol. 22, 275-280. doi: 10.1097/FBP.0b013e328345f758

Stewart, A. M., and Kalueff, A. V. (2014). The behavioral effects of acute $\Delta^{9}$. tetrahydrocannabinol and heroin (diacetylmorphine) exposure in adult zebrafish. Brain Res. 1543, 109-119. doi: 10.1016/j.brainres.2013.11.002

Szobota, S., Gorostiza, P., Del Bene, F., Wyart, C., Fortin, D. L., Kolstad, K. D., et al. (2007). Remote control of neuronal activity with a light-gated glutamate receptor. Neuron 54, 535-545. doi: 10.1016/j.neuron.2007.05.010

Tecott, L. H., and Nestler, E. J. (2004). Neurobehavioral assessment in the information age. Nat. Neurosci. 7, 462-466. doi: 10.1038/nn1225

U.S. Food and Drug Administration. (2014). FDALabel. Available at: http://www. fda.gov/ScienceResearch/BioinformaticsTools/ucm289739.htm [accessed April $13,2014]$.

Valente, A., Huang, K. H., Portugues, R., and Engert, F. (2012). Ontogeny of classical and operant learning behaviors in zebrafish. Learn. Mem. 19, 170-177. doi: $10.1101 / \mathrm{lm} .025668 .112$

Williams, L. R., Wong, K., Stewart, A., Suciu, C., Gaikwad, S., Wu, N., et al. (2012). Behavioral and physiological effects of RDX on adult zebrafish. Comp. Biochem. Physiol. C Toxicol. Pharmacol. 155, 33-38.

Wittmann, C., Reischl, M., Shah, A. H., Mikut, R., Liebel, U., and Grabher, C. (2012). Facilitating drug discovery: an automated high-content inflammation assay in zebrafish. J. Vis. Exp. 65, e4203. doi: 10.3791/4203

Wolman, M. A., Jain, R. A., Liss, L., and Granato, M. (2011). Chemical modulation of memory formation in larval zebrafish. Proc. Natl. Acad. Sci. U.S.A. 108, 1546815473. doi: 10.1073/pnas.1107156108

Wyart, C., Del Bene, F., Warp, E., Scott, E. K., Trauner, D., Baier, H., et al. (2009). Optogenetic dissection of a behavioural module in the vertebrate spinal cord. Nature 461, 407-410. doi: 10.1038/nature08323

Yokogawa, T., Marin, W., Faraco, J., Pézeron, G., Appelbaum, L., Zhang, J., et al. (2007). Characterization of sleep in zebrafish and insomnia in hypocretin receptor mutants. PLoS Biol. 5:e277. doi: 10.1371/journal.pbio.0050277

Zhdanova, I. V. (2006). Sleep in zebrafish. Zebrafish 3, 215-226. doi: 10.1089/zeb.2006.3.215

Conflict of Interest Statement: The authors declare that the research was conducted in the absence of any commercial or financial relationships that could be construed as a potential conflict of interest.

Received: 30 March 2014; accepted: 11 June 2014; published online: 24 July 2014. Citation: Bruni G, Lakhani P and Kokel D (2014) Discovering novel neuroactive drugs through high-throughput behavior-based chemical screening in the zebrafish. Front. Pharmacol. 5:153. doi: 10.3389/fphar.2014.00153

This article was submitted to Experimental Pharmacology and Drug Discovery, a section of the journal Frontiers in Pharmacology.

Copyright (c) 2014 Bruni, Lakhani and Kokel. This is an open-access article distributed under the terms of the Creative Commons Attribution License (CC BY). The use, distribution or reproduction in other forums is permitted, provided the original author(s) or licensor are credited and that the original publication in this journal is cited, in accordance with accepted academic practice. No use, distribution or reproduction is permitted which does not comply with these terms. 